\title{
Reactor performance, system reliability, instrumentation and control
}

\author{
Andreas Schumm ${ }^{1, *}$, Madalina Rabung ${ }^{2}$, Gregory Marque ${ }^{1}$, and Jary Hamalainen ${ }^{3}$ \\ ${ }^{1}$ EDF Labs Les Renardières, EDF R\&D, Avenue des Renardières, 77818 Moret sur Loing, France \\ ${ }^{2}$ Fraunhofer IZFP, Campus E3.1, 66123 Saarbrücken, Germany \\ ${ }^{3}$ VTT Technical Research Centre of Finland, Vuorimiehentie 3, Espoo, Finland
}

Received: 12 March 2019 / Accepted: 4 June 2019

\begin{abstract}
We present a cross-cutting review of three on-going Horizon 2020 projects (ADVISE, NOMAD, TEAM CABLES) and one already finished FP7 project (HARMONICS), which address the reliability of safetyrelevant components and systems in nuclear power plants, with a scope ranging from the pressure vessel and primary loop to safety-critical software systems and electrical cables. The paper discusses scientific challenges faced in the beginning and achievements made throughout the projects, including the industrial impact and lessons learned. Two particular aspects highlighted concern the way the projects sought contact with end users, and the balance between industrial and academic partners. The paper concludes with an outlook on follow-up issues related to the long term operation of nuclear power plants.
\end{abstract}

\section{Introduction}

The effective maintenance of nuclear power plants is essential for their safe operation. Maintenance ensures that the level of reliability and effectiveness of all safetyrelevant components and systems remains in accordance with design assumptions, and also that it is not adversely affected during operation [1].

Scheduling preventive and corrective maintenance operations requires an understanding of ageing mechanisms for the different components and materials used in plants, as well as a thorough and quantitative assessment of the health and reliability of safety-relevant components.

The projects addressed in this paper attempt to answer to this challenge, and cover a wide range of "safety relevant components and systems". ADVISE [2] and NOMAD [3] aim to improve quantitative Non-destructive Evaluation Techniques (NDE) to components in the primary loop (restricted to cladded components in NOMAD and to materials with complex microstructure in ADVISE) to obtain a quantitative assessment of the structural integrity of the components at hand. TEAM CABLES [4] aims to improve the understanding of ageing mechanisms on cables used in plants (specifically to the polymers used in the insulation), to model this ageing, and to devise NDE and monitoring techniques for the health assessment.

\footnotetext{
* e-mail: andreas.schumm@edf.fr
}

HARMONICS [5], the only project of the four already terminated, extends this approach to the software of computer-based I\&C safety systems.

This review is intended to be voluntarily cross-cutting, focusing on achievements, challenges and impacts of these projects rather than giving exhaustive descriptions, with an aim to identify potential follow-ups to cover the terrain not dealt with throughout these projects. We, therefore, restrict the project descriptions to brief portraits in the following paragraphs (Tab. 1).

\subsection{ADVISE}

ADVISE is an acronym for "advanced inspection of complex structured materials", and aims to advance the ultrasonic inspection of complex structured materials, for which conventional ultrasonic techniques suffer from severe performance limitations due to the micro and/or macro-structure. The most prominent examples of materials concerned are welds and cast austenitic stainless steel.

The key idea of the project is to use a-priori, modelpredicted and in-situ obtained information about the structure to be inspected in computer modelling in all stages of the inspection to obtain a step change improvement in terms of inspectable depth, defect detection and characterisation accuracy:

- during the inspection design, model-assisted optimisation of customised transducers and delay laws aims to specify the most appropriate inspection approach; 
Table 1. Key figures for concerned projects.

\begin{tabular}{llllll}
\hline Project & Duration & Funding & Lead & Partners & Framework \\
\hline ADVISE & $09 / 17-09 / 21$ & $4,2 \mathrm{ME}$ & EDF & 11 & H2020 \\
NOMAD & $06 / 17-12 / 21$ & $4,9 \mathrm{ME}$ & Fraunhofer & 10 & H2020 \\
TEAM CABLES & $09 / 17-12 / 21$ & $4,2 \mathrm{ME}$ & EDF & 13 & H2020 \\
HARMONICS & $01 / 11-01 / 15$ & $1,0 \mathrm{ME}$ & VTT & 5 & FP7 \\
\hline
\end{tabular}

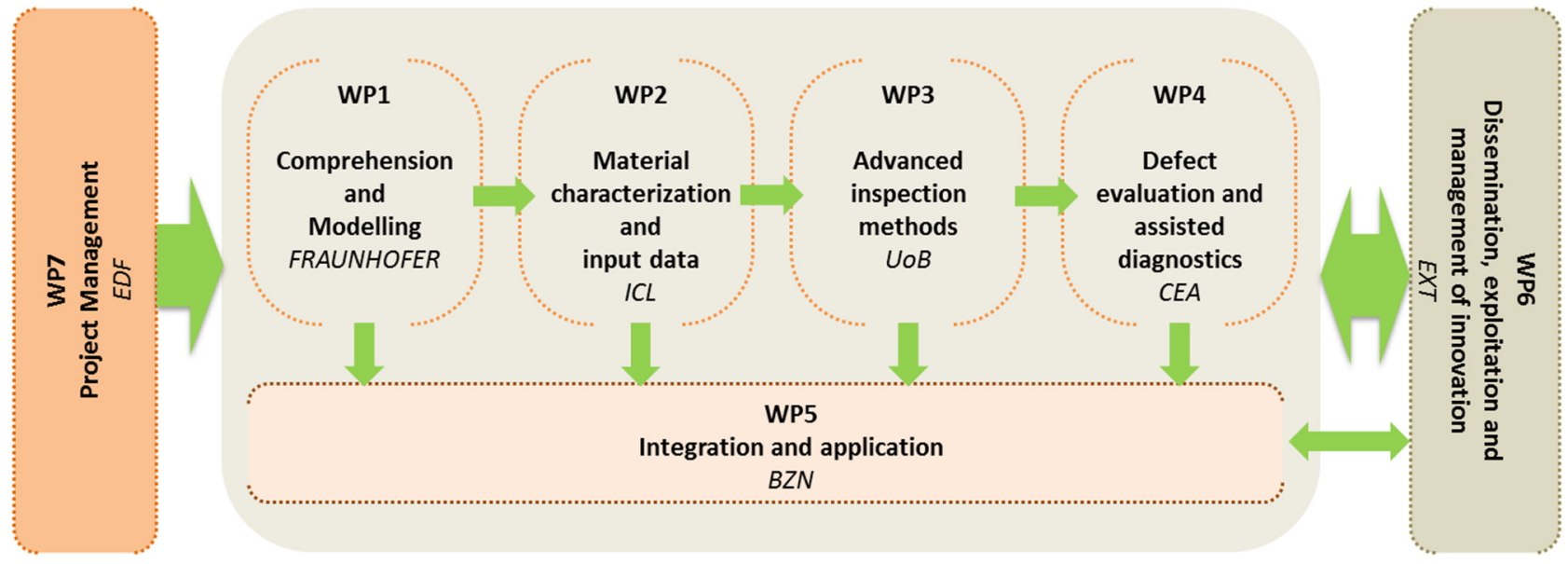

Fig. 1. ADVISE work plan.

- during the acquisition, in-situ characterisation techniques aim to acquire specific information about the structure to be inspected;

- after the acquisition, model-assisted diagnostic tools exploit the entire available information in adaptive imaging and inversion techniques.

The project admits that no single magic bullet exists, and that a number of incremental improvements need to be combined. The consortium includes industrial stakeholder, academics with specific background for the R\&D tasks, and an equipment manufacturer, as well as a distributor for rapid dissemination (Fig. 1).

\subsection{NOMAD}

NOMAD means "Nondestructive Evaluation (NDE) System for the Inspection of Operation-Induced Material Degradation in Nuclear Power Plants" and aims to develop and demonstrate an NDE approach for the quantification of neutron radiation-induced embrittlement in cladded reactor pressure vessel materials. Additionally, NOMAD focuses on the validation of the existing surveillance programs with respect to the actual vessel under LTO conditions, in terms of equivalence of radiation damage accumulation. These topics are of particular importance in terms of lifetime extension of existing operating reactors, the reactor pressure vessel
(RPV) being considered the only part of the primary loop, which cannot be replaced $[7,8]$.

A multiple scale of samples from Charpy samples, over non-cladded blocks to realistic cladded blocks, made from representative steels of eastern and western RPV design are made available in various irradiated conditions representing different realistic degradation levels. Multiple NDE technologies, including micromagnetic, electrical and ultrasound-based methods, are developed and applied to these multiple scales of samples in neutron-irradiated condition. The results are to be compared and combined across methods, samples and degradation parameters in order to define a hybrid approach and finally demonstrate it in a modular way.

For the first time, a systematic study in terms of correlation of microstructure, mechanical properties, neutron irradiation conditions and non-destructive properties is carried out on a well-characterized set of samples. The aim is not only to extend the existing database, but also to include issues such as reliability and uncertainties of the techniques as well as effects caused by material heterogeneity. Furthermore, the capabilities of the individual NDE techniques and, as result, the performance of the NDE tool regarding the future application in the field will be determined. The NOMAD consortium consists of partners with complementary expertise having common interest in the project goals: academic partners for identifying the problems in details and developing the 


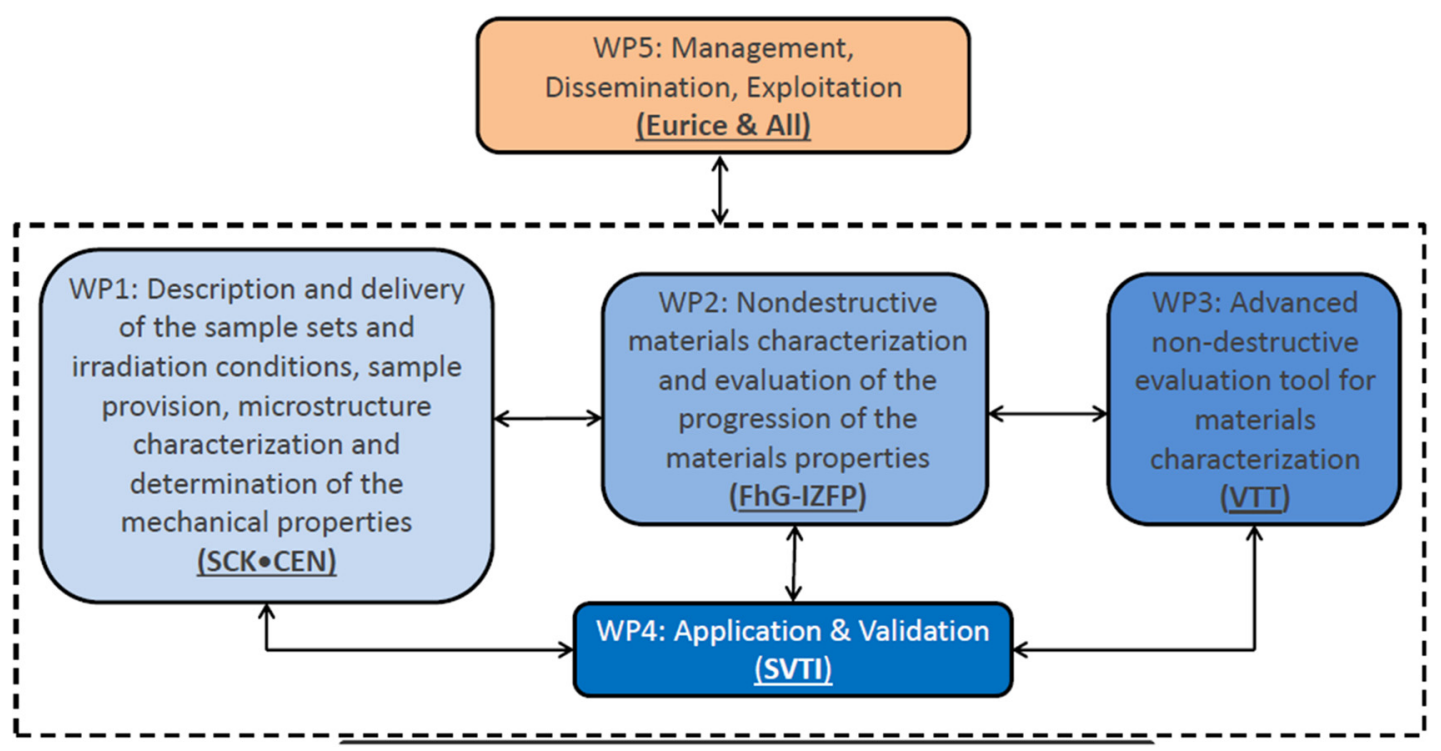

Fig. 2. NOMAD work plan.

suitable measurement methods, industrial partners guiding the developments by representing the market-needs and also industrial partners, end user group or external scientific advisory board for the validation of the needs but the solutions as well (Fig. 2).

\subsection{TEAM CABLES}

TEAM CABLES focuses on European tools and methodologies for an efficient ageing management of nuclear power plant cables and addresses the challenge of long term operation for cables - more precisely, their polymer insulation, which is subjected to aging. The sheer amount of cables in a NPP (about $1500 \mathrm{~km}$ for one nuclear unit, or twice as much for a typical 2 reactor plant) makes the replacement of cables economically unfeasible, which requires for accurate predictive models for their safe lifetime, as well as for generic tools and methods for on-site monitoring.

TEAM CABLES will develop a novel multiscale approach for more precise estimation of the cable lifetime. Cable lifetime is governed by polymer layers lifetime. A large part of the project is so dedicated to polymer science. The project will analyse the effects of irradiation and temperatures on polymers from micro- to macroscale level, in order to develop multiscale models of ageing. Ageing in normal operation conditions and accidental conditions will be addressed. The unique multi-scale and kinetic models will be integrated into a numerical tool, which will be based on the fusion of a currently used European cable management instrument with a polymer ageing modelling tool. In parallel, criteria and protocols will be proposed for onsite use of non-destructive testing techniques.

The program combines highly scientific work packages for the actual polymer ageing kinetics models with experimental work packages to obtain data throughout accelerated ageing. The consortium is comprised of stakeholders, cable manufacturers, academic partners with specific experience in polymer aging kinetics modelling, as well as applied institutes for the experimental and NDE aspects (Fig. 3).

\subsection{HARMONICS}

HARMONICS (abbreviation for Harmonised Assessment of Reliability of MOdern Nuclear I\&C Software) recognized that software can in general not be proven to be completely defect-free, and addressed the issue of reliability and safety of the computer-based systems that implement safety functions in nuclear power plants. HARMONICS had the objective to ensure well founded and up-to-date methods and data for assessing software of computer-based safety systems in Gen-II and Gen-III NPPs throughout the entire system lifecycle. It has taken advantage of the aforementioned advances to propose systematic and consistent, yet realistic and practical approaches for software assessment.

The project addressed three key issues: software verification \& validation $(\mathrm{V} \& \mathrm{~V})$, software safety justification, and quantitative evaluation of software reliability. The term "software reliability" is used as a shortcut for "software-related aspects of system reliability". The focus was mainly on I\&C systems performing category A functions (as defined by IEC 61226), which is the highest safety category in NPP. To support research activities on these three main issues, the project investigated and developed theories, techniques and tools as necessary. In addition, the feasibility of the developed approaches was experimented and demonstrated with selected case examples provided by the project participants and the end user group.

Related to the IAEA Report on Dependability Assessment of Software for Safety I\&C Systems at NPPs started in May 2014, major results from the HARMONICS project were proposed (approaches to improve confidence in functional requirements, role of formal software verification, safety justification framework). 


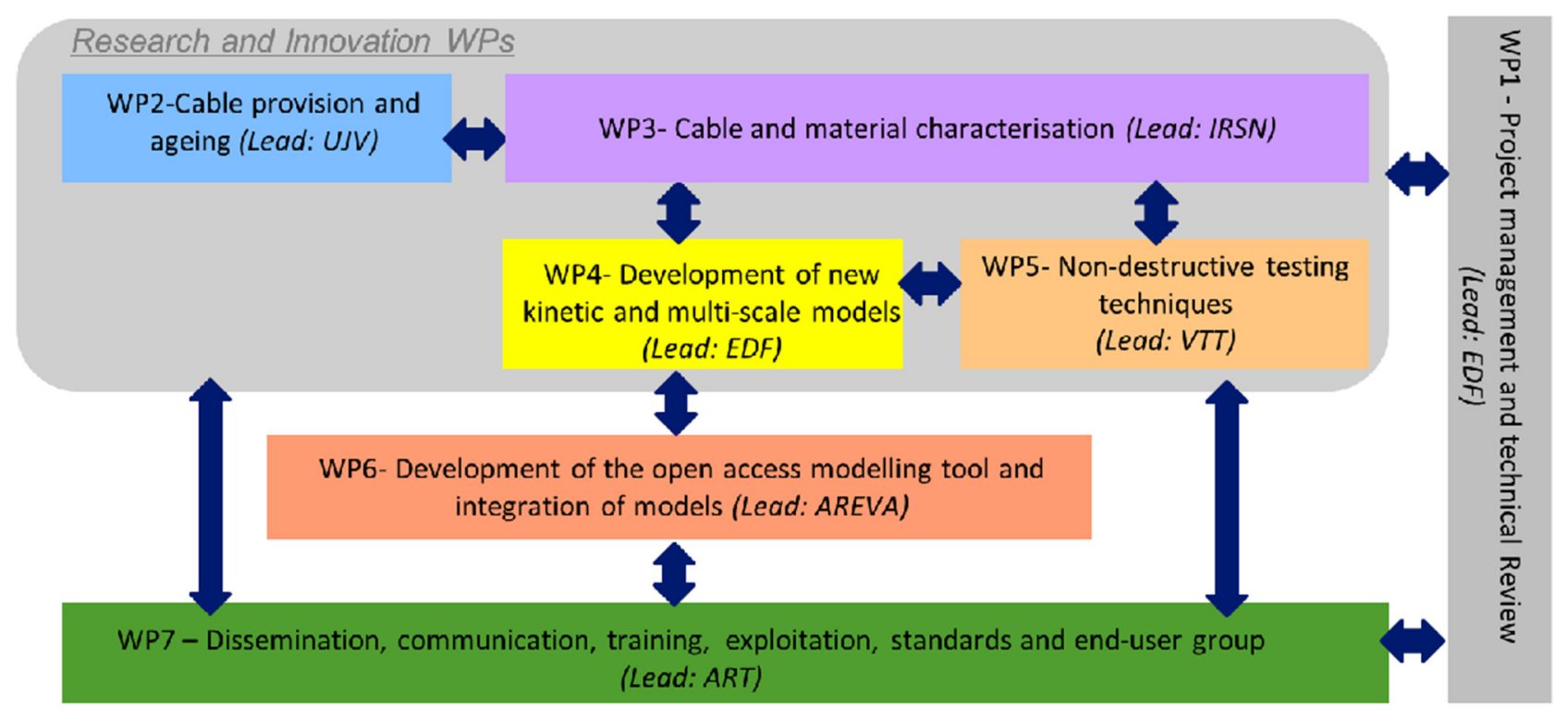

Fig. 3. TEAM CABLES work plan.

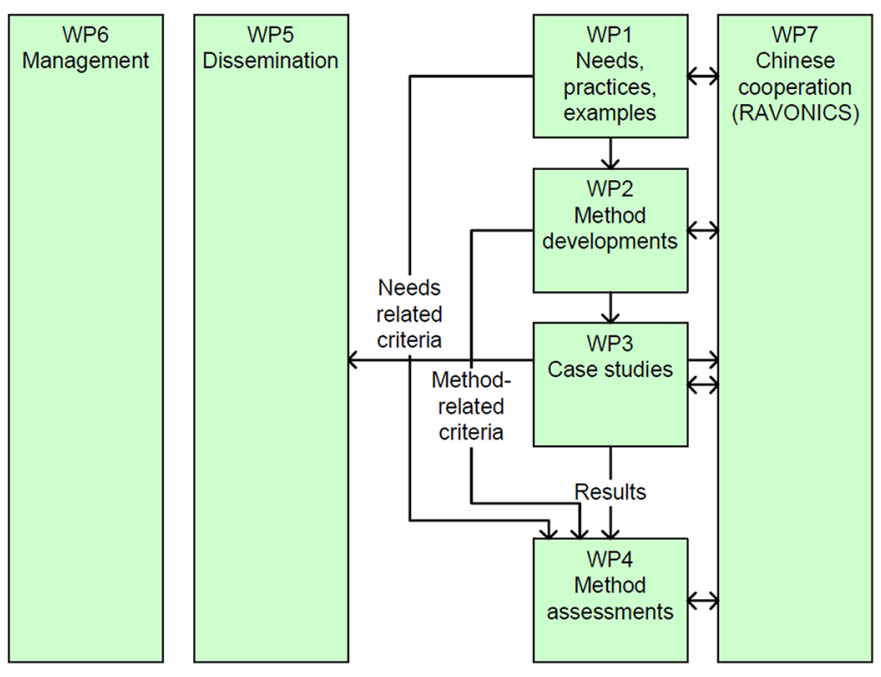

Fig. 4. HARMONICS work plan.

The consortium regrouped utilities and safety authorities and consultants, led by a multidisciplinary research organisation. As a particularity, HARMONICS had a parallel project on reliability and $\mathrm{V} \& \mathrm{~V}$ of nuclear safety I\&C software in China (Fig. 4).

\section{Challenges, achievements, impact}

\subsection{Scientific challenges and achievements}

With three out of four projects running in their second year and only one terminated, it is sensible to discuss challenges and achievements at the same time.

The principal scientific challenge faced by HARMONICS was to formally justify high to very high reliability figures for a given piece of software: it is extremely difficult to claim and formally demonstrate failure probabilities lower than $1 \mathrm{E}-4$, and moreover, no universally accepted approach for the quantitative evaluation of software reliability exists. HARMONICS answered this challenge with a safety justification framework for the software of systems implementing category A nuclear safety functions. HARMONICS created scientific deliverables covering formal verification methods, a safety justification framework, a proposed approach to quantify software reliability, and a method on complexity analysis. A comprehensive list of publications with summaries can be found on the project's website.

ADVISE, NOMAD and TEAM CABLES are funded in the frame of the section "Continually improving safety and reliability of Generation II and III reactors" of the Euratom Program 2016. The main scientific challenge of all these projects is to obtain a deeper understanding of operationinduced degradation mechanisms. This will be carried out by applying innovative NDE methods in ADVISE and NOMAD, and will be used to develop NDE methods in TEAM CABLES.

ADVISE established the scientific challenges early on in the project in the first technical delivery in the form of a state-of-the-art report. The project considers this as a good means to take a snapshot at the start of the project, which shall be used at the end of the project to measure the achievements. A major challenge for the project concerns the experimental non-destructive (as opposed to destructive laboratory analysis) characterization of material microstructure in situ, the prediction of micro- and macrostructures in weld models, and the ability of fast ultrasound simulation models to take material microstructure into account.

NOMAD's main scientific challenge is the quantification of neutron irradiation-induced embrittlement of RPV steels independent on the austenitic cladding, combining information from multiple non-destructive evaluation techniques. To this, signals originated from the cladding 
must be separated from the signals obtained from the base material. Another challenge is to produce irradiated cladded blocks similar to the real operating RPV and after that to compare the non-destructively detected material properties with those detected on irradiated Charpy samples. To this, NDE methods based on different physical principles have been developed and successfully tested on neutron-irradiated Charpy samples and thermally aged cladded blocks.

TEAM CABLES faces multiple scientific challenges related to polymer ageing, which are in part covered by three $\mathrm{PhD}$ collaborations with academic partners. The overall ambition of TEAM CABLES is to allow NPP operators to improve their capacity to safely manage the lifetime of cables and thereby contribute to ensuring the lifetime extension of NPPs to $60-80$ yr. To achieve this, a radically new way to predict the lifetime of cables (in terms of mechanical, physical and electrical parameters) is developed, using much more precise information about material composition and more relevant methods for analysing the data based on multi-scale studies of the materials.

\subsection{Industrial impact}

Shortly before the end of the HARMONICS project, the IAEA had started the development of a technical report on the Dependability Assessment of Software for Safety I\&C Systems at NPPs. Several members of the HARMONICS project were part of the expert team that drafted the report, and some major results from the project were ultimately integrated into this report (approaches to improve confidence in functional requirements, role of formal software verification, safety justification framework). The research problems and the results were also disseminated in the end user workshops during the project.

In the short term, TEAM CABLES and NOMAD intend to achieve industrial impact through a series of end user workshops, and a closing symposium. Both projects will deliver tools capable of delivering additional substantial information regarding the degradation parameters used for the assessment of LTO, non-destructively, fast and reducing the consumed surveillance material. TEAM CABLES will organize a training workshop for NPP operators and researchers on the developed tool. ADVISE takes a different approach, relying on the acquisition system manufacturer and the distributor of the CIVA software package to achieve rapid industrial impact. NOMAD and ADVISE realize that any novel NDE procedure will ultimately go through qualification, which is difficult to anticipate at this early stage.

In the medium term, these projects shall provide the background for robust national and EU strategies in the field of nuclear reactor safety in order to further improve the safety of RPVs in Europe and worldwide through increased resistance of safety relevant equipment. In the long term, results of these projects should strengthen the competitiveness and growth of companies by developing innovations meeting the needs of European and global markets, and where relevant, by delivering such innovations to the markets.

\subsection{End user implication}

Horizon 2020 focuses on dissemination, which clearly emerges in all ongoing projects. All three projects have designated dissemination work packages. TEAM CABLES pushes this idea particularly far, with a summer school, two end user workshops, a training workshop for NPP operators and researchers as well as a final symposium. ADVISE and NOMAD will hold at least one joint public symposium. To ensure the industrial applicability of models and tools developed in all those projects, end user groups composed of external advisors have been set up, with the main goal to assess the developed models and tools during and by the end of the project.

HARMONICS, which was funded by FP7, held two end user workshops in order to establish and maintain a link with stakeholders. All projects set up public web sites with detailed descriptions of the projects and their publications $[2-5]$.

\subsection{Academic involvement}

TEAM CABLES collaborates with the University of Bologna and ENSAM Paris, with a total of three PhDs. They will work on the development and validation of a kinetics model for polymer aging, and the use of the output of the kinetics models in multiscale models to predict mechanical, physical and electrical parameters. ADVISE and NOMAD employ several young researchers with first time contact to the nuclear industry. Four researchers with a $\mathrm{PhD}$ degree and two $\mathrm{PhD}$ students worked in HARMONICS.

\section{Lessons learnt}

A common challenge shared by all projects concerns the capitalization of achievements made. TEAM CABLES realized this already at the proposal stage and centres its capitalization effort around a software tool as a federating item. For ADVISE, the situation is more challenging, as a commercial software platform has been chosen to become the target of the various work-packages, which is inherently more complex and needs to comply with more requirements and restrictions. The consortium held a dedicated two day training session to address this difficulty. During the development stage, a simpler rapid prototyping tool is thus used before integration into the commercial software. Due to the nature of the HARMONICS project, a natural way to capitalize achievements was via an IAEA Safety Series publication [6].

For an experiment-centric project such as NOMAD, which deals with the characterisation of changes of the materials properties due to neutron irradiation, the characterisation of same samples before and after irradiation connected with samples irradiation beyond periodical safety reviews revealed to be a challenging issue. Such a procedure has never been performed before and turned out to require an extremely extensive preparation.

All projects were confronted with the issue of how to extend the scope of their work beyond western nuclear technology. NOMAD was able to secure a comprehensive 
range of neutron irradiated samples for eastern and western base and weld material, as well as non-irradiated samples from different RPV steels. ADVISE has access to Russian VVER type reactor samples through its partner UJV, who is also member of the TEAM CABLES project. HARMONICS made an attempt to enlarge its scope by teaming up with a parallel Chinese project, although the added value of this collaboration turned out to be disappointing.

\section{Conclusions and follow-up issues}

For the larger part of the currently operating generation, 2 plants with an initial design life of $40 \mathrm{yr}$, the lifetime extension to 60 years has become economically viable, and is partly due to the increased capital cost of generation $3+$ reactors. The long term operation of these plants has raised issues, which are at the origin of the three ongoing H2020 projects discussed in this paper. For many components of these plants, NDE has often been designed as an afterthought, rather than being an integral part of the design. This lesson has been learnt, and leads to three interesting paradigm changes:

- continuous monitoring of the structural health of components has demonstrated its added value in other industries (such as aviation/aerospace) as a complement to in-service inspections at programmed intervals, and is progressively making its way into the nuclear industry;
- ageing models, fed with data from continuous monitoring and in service inspections, allow for predictive maintenance (as opposed to scheduled maintenance). The question of how to aggregate and use such data has led to the development of digital replica of components;

- inspection-oriented design, already well-established in instrumentation and control, has to be considered at manufacture and for replacement components.

\section{References}

1. Maintenance of Nuclear Power Plants, IAEA Safety Series No. 50-OG-07, 1990

2. ADVISE public website, https://www.advise-h2020.eu/

3. NOMAD public website, https://nomad-horizon2020.eu/

4. TEAM CABLES public website, https://www.team-cables.eu/

5. HARMONICS public website, http://harmonics.vtt.fi/ (see D5.6 for publications and D5.5 for end user workshop proceedings)

6. Instrumentation and Control Systems and Software Important to Safety for Research Reactors, IAEA Safety Guide SSG-38, 2015

7. The Economics of Long-term Operation of Nuclear Power Plants (Nuclear Energy Agency and Organisation for Economic co-operation and development, 2012)

8. A. Ballesteros, R. Ahlstrand, C. Bruynooghe, U. von Estorff, L. Debarberis, The role of pressure vessel embrittlement in the long term operation of nuclear power plants, Nucl. Eng. Des. 243, 63 (2012)

Cite this article as: Andreas Schumm, Madalina Rabung, Gregory Marque, Jary Hamalainen, Reactor performance, system reliability, instrumentation and control, EPJ Nuclear Sci. Technol. 6, 43 (2020) 